the power series for $(\bar{a}-b z)^{-1}$, however, converges as previously required, hence we have that $U(\sigma) z U\left(\sigma^{-1}\right)=(a z-\bar{b}) /(\bar{a}-b z)=\sigma^{-1} z$.

\title{
REFERENCES
}

1. G. W. Mackey, Imprimitivity for representations of a locally compact group. I, Proc. Nat. Acad. Sci. U.S.A. 35 (1949), 537-545.

2. - Unitary representations of group extensions. I, Acta Math. 99 (1958), 265-311.

3. R. J. Blattner, On induced representations. II. Infinitesimal induction, Amer. J. Math. 83 (1961), 499-512.

4. V. Bargmann, Irreducible unitary representations of the Lorenz group, Ann. of Math. (2) 48 (1947), 568-640.

ILLINOIS INSTITUTE OF TECHNOLOGY

\section{ON WANDERING SUBSPACES FOR UNITARY OPERATORS}

J. B. ROBERTSON ${ }^{1}$

Let $V$ be a unitary operator on a complex Hilbert space $H . X$ is said to be a wandering subspace for $V$ if it is a subspace of $H$ such that $V^{m}(X) \perp V^{n}(X)$ for all $m \neq n$. The purpose of this note is to study the relation between two wandering subspaces $X$ and $Y$ satisfying $\sum_{k=-\infty}^{\infty} V^{k}(X) \subseteq \sum_{k=-\infty}^{\infty} V^{k}(Y)$.

THEOREM 1. Let $X$ and $Y$ be wandering subspaces for a unitary operator $V$ such that:

(a) $\sum_{k=-\infty}^{\infty} V^{k}(X) \subseteq \sum_{k=-\infty}^{\infty} V^{k}(Y)$,

(b) $\operatorname{dim}(X)=\operatorname{dim}(Y)<\infty$.

Then $\sum_{k=-\infty}^{\infty} V^{k}(X)=\sum_{k=-\infty}^{\infty} V^{k}(Y)$.

Proof. Let $x_{1}, \cdots, x_{n}$ and $y_{1}, \cdots, y_{n}$ be orthonormal bases for $X$ and $Y$, respectively. Since $x_{i} \in \sum_{k=-\infty}^{\infty} V^{k}(Y)$ we have

$$
\begin{aligned}
x_{i}= & \sum_{r=1}^{n} \sum_{k=-\infty}^{\infty} a_{i r k} V^{k}\left(y_{r}\right), \quad a_{i r k}=\left(x_{i}, V^{k}\left(y_{r}\right)\right), \\
& \sum_{r=1}^{n} \sum_{k=-\infty}^{\infty}\left|a_{i r k}\right|^{2}<\infty, \quad i=1, \cdots, n .
\end{aligned}
$$

It follows that

Received by the editors October 30, 1963.

${ }^{1}$ This research was supported in part by National Science Foundation Grant No. GP 2234 while the author was at Cornell University. 


$$
\delta_{m 0} \delta_{i j}=\left(x_{i}, V^{m}\left(x_{j}\right)\right)=\sum_{r=1}^{n} \sum_{k=-\infty}^{\infty} a_{i r k} \bar{a}_{j r k-m}
$$

Since $\sum_{k=-\infty}^{\infty}\left|a_{i r k}\right|^{2}<\infty$, there exists a function $f_{i r}(\cdot) \in L(0,2 \pi)$ such that $f_{i r}(\theta) \sim \sum_{k=-\infty}^{\infty} a_{i r k} e^{i k \theta}$. (1) may now be restated as

$$
\sum_{r=1}^{n} f_{i r}(\theta) \vec{f}_{j r}(\theta)=\delta_{i j} \text { a.e. (Leb.), } i, j=1, \cdots, n .
$$

By the usual theory of $n \times n$ matrices, $\left(1^{\prime}\right)$ is equivalent to

$$
\sum_{i=1}^{n} \bar{f}_{i r}(\theta) f_{i s}(\theta)=\delta_{r s} \text { a.e. (Leb.), } \quad r, s=1, \cdots, n \text {. }
$$

$\left(2^{\prime}\right)$, in turn, may be restated in terms of the coefficients as

$$
\delta_{m 0} \delta_{r \varepsilon}=\sum_{i=1}^{n} \sum_{k=-\infty}^{\infty} \bar{a}_{i r k} a_{i s k-m}
$$

Now let $Q$ be the projection operator onto the space $\sum_{k=-\infty}^{\infty} V^{k}(X)$. Since $\left(y_{r}, V^{k}\left(x_{i}\right)\right)=\bar{a}_{i r-k}$, we have

$$
Q\left(y_{r}\right)=\sum_{i=1}^{n} \sum_{k=-\infty}^{\infty} a_{i r-k} V^{k}\left(x_{i}\right), \quad r=1, \cdots, n .
$$

From (2) we obtain

$$
\left\|Q\left(y_{r}\right)\right\|^{2}=\sum_{i=1}^{n} \sum_{k=-\infty}^{\infty}\left|a_{i r-k}\right|^{2}=1, \quad r=1, \cdots, n .
$$

Therefore, $\left\|y_{r}\right\|=\left\|Q\left(y_{r}\right)\right\|$ which implies $y_{r}=Q\left(y_{r}\right) \in \sum_{k--\infty}^{\infty} V^{k}(X)$. Thus $\sum_{k=-\infty}^{\infty} V^{k}(X)=\sum_{k=-\infty}^{\infty} V^{k}(Y)$. Q.E.D.

Theorem 1 is clearly false if $\operatorname{dim}(Y)=\infty$, but the following corollary, due to Halmos [1, Lemma 4], is true for all dimensions.

CoROllaRY. Let $X$ and $Y$ be wandering subspaces for a unitary operator $V$ such that $\sum_{k=-\infty}^{\infty} V^{k}(X) \subseteq \sum_{k=-\infty}^{\infty} V^{k}(Y)$. Then $\operatorname{dim}(X)$ $\leqq \operatorname{dim}(Y)$.

Proof. If the dimension of $Y$ is not finite then, since $X \subseteq \sum_{k=-\infty}^{\infty} V^{k}(Y), \operatorname{dim}(X) \leqq \operatorname{dim}\left(\sum_{k=-\infty}^{\infty} V^{k}(Y)\right)=\operatorname{dim}(Y)$ as desired. Next let $\operatorname{dim}(Y)=n<\infty$ and suppose $\operatorname{dim}(X)>n$. If $x_{1}, \cdots$, $x_{n+1}$ are orthonormal vectors in $X$ and if $\tilde{X}$ is the space spanned by $x_{1}, \cdots, x_{n}$, then $\sum_{k=-\infty}^{\infty} V^{k}(\tilde{X})=\sum_{k--\infty}^{\infty} V^{k}(Y)$ !by Theorem 1. Thus $x_{n+1} \in\left(\sum_{k=-\infty}^{\infty} V^{k}(\tilde{X})\right)^{\perp} \cap\left(\sum_{k=-\infty}^{\infty} V^{k}(Y)\right)=\{0\}$ |which contradicts the assumption that $x_{n+1}$ is normal. Thus $\operatorname{dim}(X) \leqq n$. Q.E.D. 
It follows from the corollary, of course, that if $\sum_{k=-\infty}^{\infty} V^{k}(X)$ $=\sum_{k=-\infty}^{\infty} V^{k}(Y)$, then $\operatorname{dim}(X)=\operatorname{dim}(Y)$. The final theorem shows that finite-dimensional wandering subspace may be increased to the maximum.

Theorem 2. Let $X$ and $Y$ be wandering subspaces for a unitary operator $V$ such that:

(a) $\sum_{k=-\infty}^{\infty} V^{k}(X) \subseteq \sum_{k=-\infty}^{\infty} V^{k}(Y)$,

(b) $\operatorname{dim}(Y)<\infty$.

Then there exists a wandering subspace $\tilde{X}$ such that:

(1) $X \subseteq \tilde{X}$

(2) $\sum_{k=-\infty}^{\infty} V^{k}(\tilde{X})=\sum_{\mathbf{k}=-\infty}^{\infty} V^{k}(Y)$.

Proof. Let $x_{1}, \cdots, x_{m}$ and $y_{1}, \cdots, y_{n}$ be orthonormal bases for $X$ and $Y$, respectively, and suppose $m<n$. As before, let

$$
\begin{aligned}
x_{i} & =\sum_{r=1}^{n} \sum_{k=-\infty}^{\infty} a_{i r k} V^{k}\left(y_{r}\right), \\
f_{i r}(\theta) & \sim \sum_{k=-\infty}^{\infty} a_{i r k} e^{i k \theta}, \quad i=1, \cdots, m, r=1, \cdots, n .
\end{aligned}
$$

Then (cf. $\left.\left(1^{\prime}\right)\right) \boldsymbol{f}_{i}(\theta)=\left(f_{i 1}(\theta), \cdots, f_{i n}(\theta)\right), i=1, \cdots, m$, are orthonormal vectors in $C^{n}$ for almost all $\theta$. We can extend this to an orthonormal basis of $C^{n},\left(f_{1}(\theta), \cdots, f_{n}(\theta)\right)$, and this can be done measurably. (E.g., apply the Gram-Schmidt process to $\left(f_{1}(\theta), \cdots, f_{m}(\theta)\right.$, $\left.e_{1}, \cdots, e_{n}\right)$, where $\left.e_{i}=\left(\delta_{i j}\right)_{j=1}^{n}.\right)$ Since $f_{i}(\theta)$ is normal almost everywhere, its component functions $f_{i r}(\cdot)$ are in $L_{2}(0,2 \pi)$. Let, therefore,

$$
f_{i r}(\theta) \sim \sum_{k=-\infty}^{\infty} a_{i r k} e^{i k \theta}, \quad i, r=1, \cdots, n .
$$

Then, as in the proof of Theorem 1 , the space $\tilde{X}$ spanned by the orthonormal vectors

$$
x_{i}=\sum_{r=1}^{n} \sum_{k=-\infty}^{\infty} a_{i r k} V^{k}\left(y_{r}\right), \quad i=1, \cdots, n,
$$

has the desired properties. Q.E.D.

The following example shows that the assumption $\operatorname{dim}(Y)<\infty$ is essential for Theorem 2 .

EXAMPLE. Let $y_{1}, y_{2}, \cdots$ be a complete orthonormal set of vectors for a wandering subspace $Y$ of a unitary operator $V$. Let $l_{2}=\left\{a=\left(a_{1}, a_{2}, \cdots\right): \sum_{k=1}^{\infty}\left|a_{k}\right|^{2}<\infty, a_{k}\right.$ complex numbers $\}$, and let 


$$
f_{i r}(\theta)=\left\{\begin{array}{ll}
\delta_{i r} & \text { if } 0 \leqq \theta \leqq \pi \\
\delta_{i+1 r} & \text { if } \pi<\theta \leqq 2 \pi
\end{array} \sim \sum_{k=-\infty}^{\infty} a_{i r k} e^{i k \theta} .\right.
$$

Then $f_{i}(\theta)=\left(f_{i r}(\theta)\right)_{r=1}^{\infty}$ for $0 \leqq \theta \leqq 2 \pi$ are orthonormal vectors in $l_{2}$. Thus the space $X$ spanned by the orthonormal vectors

$$
x_{i}=\sum_{r=1}^{\infty} \sum_{k=-\infty}^{\infty} a_{i r k} V^{k}\left(y_{r}\right), \quad i=1,2, \cdots
$$

is a wandering subspace contained in $\sum_{k=-\infty}^{\infty} V^{k}(Y)$. $X$ cannot be extended as in Theorem 2 since this would imply the existence of a function $f:[0,2 \pi] \rightarrow l_{2}$ such that $(f(\theta), f(\theta))=1$ almost everywhere and that $\left(f(\theta), f_{i}(\theta)\right)=0, i=1,2, \cdots$, almost everywhere. But $f_{i}(\theta)$, $i=1,2, \cdots$, span $l_{2}$ for $\theta$ between 0 and $\pi$. On the other hand, if

$$
\begin{aligned}
& f_{r}(\theta)=\left\{\begin{array}{ll}
0 & \text { if } 0 \leqq \theta \leqq \pi \\
\delta_{1 r} & \text { if } \pi<\theta \leqq 2 \pi
\end{array} \sim \sum_{k=-\infty}^{\infty} b_{r k} e^{i k \theta},\right. \\
& f(\theta)=\left(f_{r}(\theta)\right)_{r=1}^{\infty},
\end{aligned}
$$

then

$$
x=\sum_{r=1}^{\infty} \sum_{k=-\infty}^{\infty} b_{r k} V^{k}\left(y_{r}\right) \in \sum_{k=-\infty}^{\infty} V^{k}(Y)
$$

is clearly orthogonal to $\sum_{k=-\infty}^{\infty} V^{k}(X)$, since $f(\theta)$ is orthogonal to $f_{r}(\theta), r=1,2, \cdots$. Thus $\sum_{k=-\infty}^{\infty} V^{k}(X) \neq \sum_{k=-\infty}^{\infty} V^{k}(Y)$.

\section{REFERENCE}

1. P. Halmos, Shifts on Hilbert space, J. Reine Angew. Math. 208 (1961), 102-112.

INDIANA UNIVERSITY AND

CORNELl University 